\title{
AC 2008-2024: USING MICROTUBULES TO ILLUSTRATE POLYMER PROPERTIES
}

\section{Yoli Jeune, University of Florida}

Yoli Jeune is currently a $\mathrm{PhD}$ candidate at the Department of Materials Science and Engineering of the University of Florida. She has received a Bachelors degree in Clinical Laboratory Sciences (1999) and a Masters degree in Secondary Science Education with a concentration in Biology (2002) from the University of South Florida. She worked for 3.5 years at the Hillsborough County School District in Florida teaching Biology and Chemistry to High School students. She is a recipient of the McKnight Doctoral Fellowship, Alfred P. Sloan, and Alliance for Graduate Education and the Professoriate fellowships.

\section{Henry Hess, University of Florida}

Henry Hess is currently an assistant professor at the Department of Materials Science and Engineering of the University of Florida. He received a diploma in physics from the Technical University Berlin in 1996, and obtained his Dr rer. nat. (summa cum laude) in experimental physics from the Free University of Berlin in 1999 under the guidance of Ludger Woeste. His postdoctoral studies were conducted from 2000 to 2002 at the Department of Bioengineering, University of Washington, where he also served as a research assistant professor (2002-2005). He received the Wolfgang Paul Award of the German Society for Mass Spectrometry (2000) and, together with his postdoctoral mentor Viola Vogel, the Philip Morris Forschungspreis (2005). 


\title{
Using microtubules to illustrate polymer properties
}

\begin{abstract}
Microtubules are a biopolymer, which assembles in vitro within minutes via noncovalent interactions from thousands of tubulin proteins at a temperature of 37 degrees Celsius. The large size ( $25 \mathrm{~nm}$ in diameter and several micrometers in length) and stiffness of these tubular, hollow polymers enables the imaging of individual, fluorescently labeled microtubules by fluorescence microscopy. We have utilized microtubules to create a stimulating laboratory, for undergraduate students which illustrates basic polymer concepts using commercially available compounds.
\end{abstract}

By imaging and analyzing a population of microtubules, students can directly determine molecular weight distributions and the degree of polymerization. Polymerization parameters, such as initial monomer concentration, temperature, and polymerization time, as well as postpolymerization processing conditions (such as shearing) can be varied, and their effect on the microtubule population can be directly observed.

Based on the assessment of the first group of students conducting this laboratory, we propose that a microtubule-based laboratory is a valuable addition to the curriculum of MSE and BME students specializing in polymers and biomaterials, since it enables striking demonstrations of polymer science and bioengineering principles.

\section{Introduction}

At our institution, a 3-credit, semester long, senior level course "Physical Properties of Polymers" is accompanied by a laboratory section. In this section, an introductory polymerization laboratory where the students polymerized Nylon 6,6 and Polymethylmethacrylate (PMMA) is followed by five laboratories focusing on rheology, surface energy, viscosity, gel permeation chromatography (GPC), and mechanical properties.

Based on our extensive experience ${ }^{1-3}$ in working in vitro with microtubules (biopolymer structures which self-assemble from the protein tubulin) we perceived an opportunity to design a novel laboratory focused on the illustration of basic polymer properties using microtubules as examples. We primarily aimed to enhance the students' intuitive grasp of concepts such as molecular weight distribution, Brownian motion, and post-polymerization processing. Due to the increasing interest of engineers in biological systems, this laboratory would also complement the traditional study of synthetic polymers with the investigation of a biopolymer having central functions in eukaryotic cells.

Microtubules are a unique polymer for our purposes due to their large size ${ }^{4,5}$. With a length of several micrometers and a diameter of $25 \mathrm{~nm}$ these tubular structures are thousand-fold larger compared to synthetic linear polymer chain such as polyethylene. Conjugation of fluorescent molecules to the tubulin subunits at a ratio of approximately one-to-one results in bright structures which can be readily imaged with a modern fluorescence microscope. Since the spatial resolution of the optical microscope is on the order of $200 \mathrm{~nm}$, the length of individual 
microtubules in a population can be determined accurately. Furthermore, the parameters of the polymerization process (proceeding at a temperature of $37^{\circ} \mathrm{C}$ on the timescale of minutes and using water as solvent) can be varied and their effect on the microtubule population can be directly observed.

\section{Microtubules: A Brief Introduction}

Microtubules are biopolymers found naturally in the cell. Microtubules are a central part of the cytoskeleton and provide structural support as well as a "highway" system for kinesin and other molecular motors to travel. Microtubules assemble via noncovalent interactions from several thousands of tubulin subunits. Each tubulin subunit is a protein dimer consisting of an alpha and a beta tubulin, as shown in the Figure 1 below. The tubulin dimers form linear protofilaments and the protofilaments assemble to form long, hollow rods. The rods have a diameter of $25 \mathrm{~nm}$ and lengths of several micrometers. Microtubules are in a dynamic equilibrium between polymerization and depolymerization ${ }^{6}$. However, by diluting a tubulin/microtubule solution into a buffer solution containing the drug paclitaxel (previously known as taxol) ${ }^{7}$, microtubules can be stabilized against depolymerization and a snapshot of the dynamic microtubule population directly prior to stabilization is obtained ${ }^{8}$.
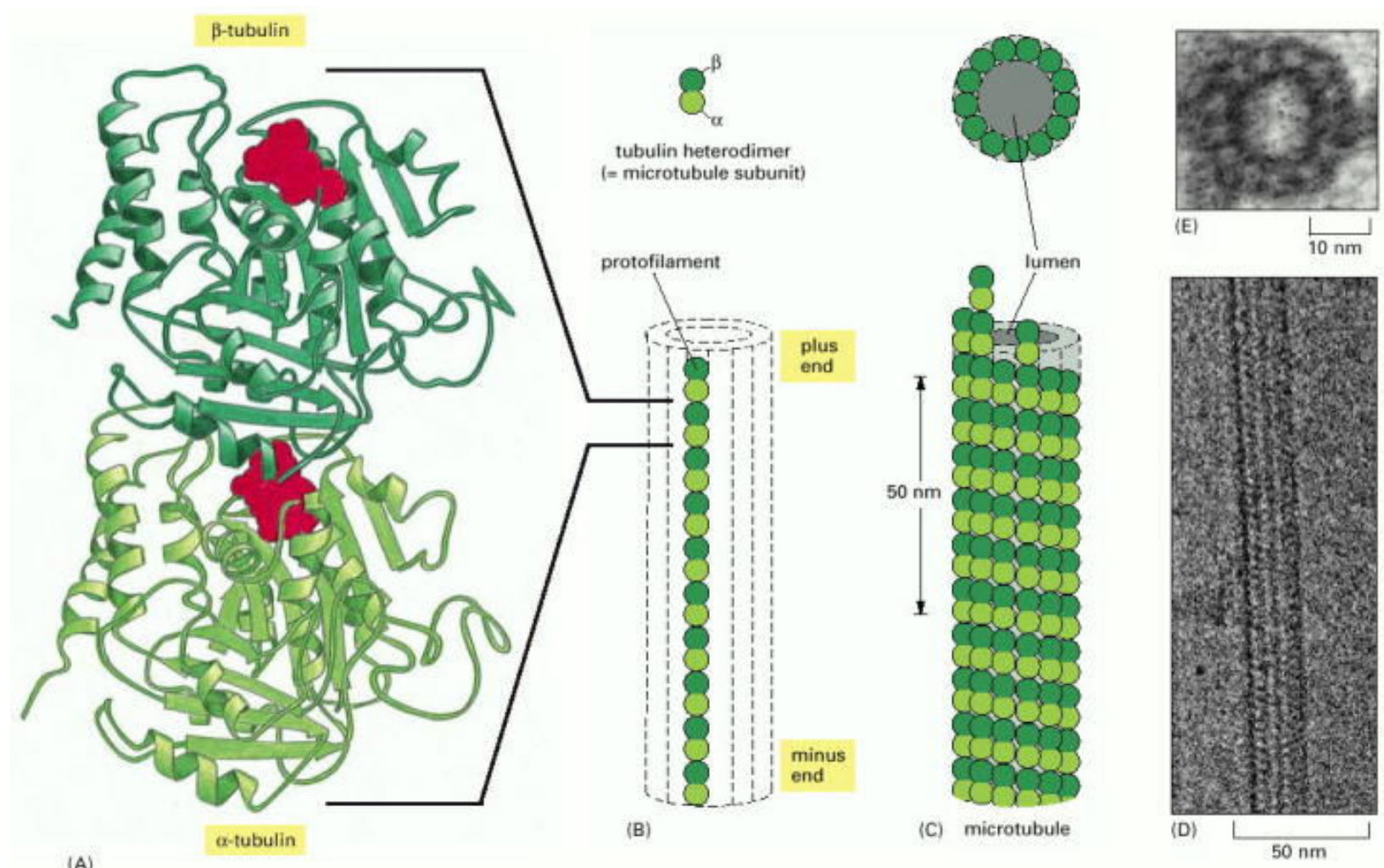

Figure 1: Structure of a microtubule and its subunits. (a) The $\alpha$ and $\beta$ tubulin monomers come together to form the subunit of a protofilament. Each monomer has a GTP molecule bound to it. (b) The protofilament assembled from tubulin dimers. (c) Microtubules are rigid, hollow cylinders consisting of approximately 13 protofilaments. (d) \& (e) Electron microscopy images of a microtubule. From Alberts et al.: "Molecular Biology of the Cell" 4. 


\section{Laboratory Description}

The materials and equipment were laid out for the students. Upon entering the laboratory, the teaching assistant had a quick pre-lab discussion with the students. After the pre-lab discussion, the students followed the procedures with minimal help from the teaching assistant.

$\underline{\text { Method }}$

First the students polymerized the microtubules; the two parameters varied for polymerization were time and temperature. A water bath set at $37^{\circ} \mathrm{C}$ was used for a high temperature polymerization; for low temperature polymerization, the students allowed the monomer to sit at room temperature. The room temperature sample was allowed to polymerize for 20 minutes, while the $37^{\circ} \mathrm{C}$ samples were varied at 2,10 and 20 minutes. For post-polymerization processing the students subjected the microtubules (polymerized at $37^{\circ} \mathrm{C}, 20$ minutes) to shearing. The students removed two $5 \mu \mathrm{L}$ aliquots and sheared one aliquot once through a $30 \mathrm{G}$ needle and sheared the second aliquot twice through the needle.

\section{$\underline{\text { Results }}$}

After the allotted time for polymerization, the students stabilized the microtubules by transferring the polymers to a pH-buffered paclitaxel solution. The students then imaged the microtubules under an epi-fluorescent microscope. The Nikon TE2000 epi-fluorescence microscope was equipped with a $100 \mathrm{~W}$ mercury lamp, a cooled camera (Andor iXon DU885LC EMCCD) and a 100x oil objective lens (N.A. 1.33). Excitation light of appropriate wavelength was supplied to the samples using suitable filters. The shutter (Lambda SC, Sutter Instruments) and the camera were controlled using a LabView program. The microtubules were exposed to light for $0.2 \mathrm{~s}$ every $200 \mathrm{~ms}$. Students captured 5-10 images per sample and saved these as Tiff files for further analysis. Due to the large size of the polymers and the fluorescent dye on the tubulin proteins, the images could be easily seen by the students. A sample of the images is shown in Figure 2, below.

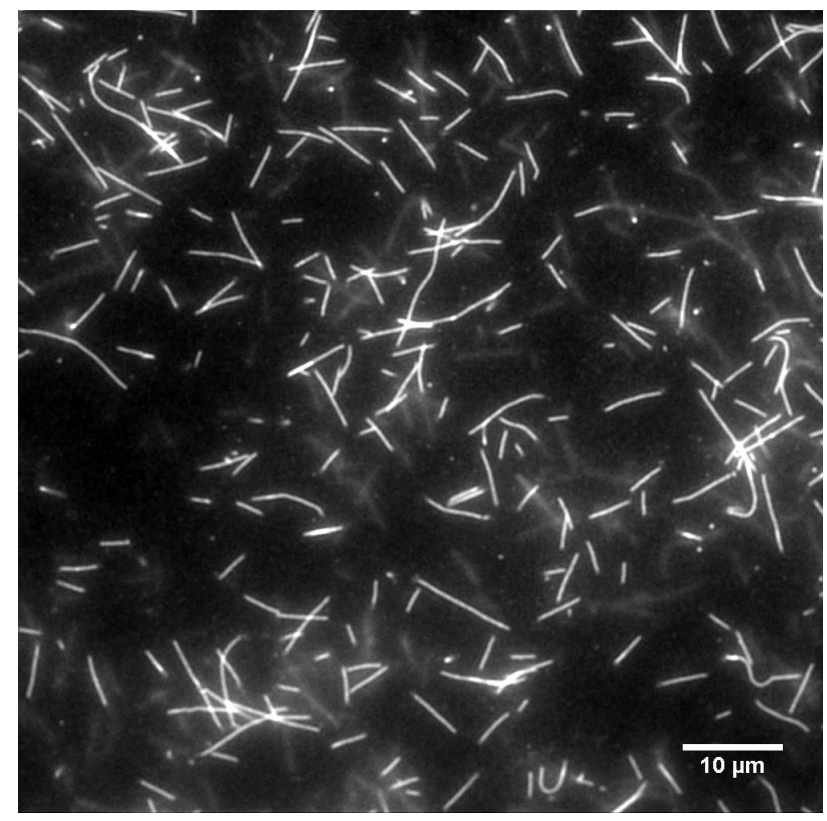

Figure 2: Fluorescent image of microtubules adhering to a coverslip surface. The microtubules were polymerized at $37^{\circ} \mathrm{C}$ for 10 minutes. 


\section{$\underline{\text { Analysis }}$}

The imaging analysis software "Image J" was used for data analysis of the images captured. Image $\mathrm{J}$ is freely available for download at http://rsb.info.nih.gov/ij/. With Image $\mathrm{J}$, the students were able to count about 500 individual microtubules and measure their lengths. From the raw data, they were able to construct a number distribution and length distribution to test various parameters of this experiment. The length of the microtubules is closely proportional to its molecular weight since their diameter is rather constant along the length of the microtubule ${ }^{9}$. Therefore the length distributions are a good representation of the weight distributions. The students were able to calculate weight-average and number-average molecular weights, or lengths in our case, of the polymers. Table 1 summarizes the length measurements for each polymerization setting.

Table 1: Averages and Standard Deviations of Length Measurements for Each Sample

\begin{tabular}{|c|c|c|c|c|c|}
\hline Sample & $\begin{array}{c}\text { Temperature } \\
\left({ }^{\circ} \mathrm{C}\right)\end{array}$ & Time $(\mathrm{min})$ & Shearing & $\begin{array}{c}\text { Number average } \\
\text { length }(\mu \mathrm{m})\end{array}$ & $\begin{array}{c}\text { Standard } \\
\text { deviation }(\mu \mathrm{m})\end{array}$ \\
\hline MT100-A & 25 & 20 & No & 7.22 & 3.53 \\
\hline MT100-B & 37 & 20 & No & 5.42 & 2.93 \\
\hline MT100-C & 37 & 10 & No & 4.04 & 2.56 \\
\hline MT100-D & 37 & 2 & No & 4.49 & 2.63 \\
\hline MT100-B1 & 37 & 20 & Once & 4.32 & 2.91 \\
\hline MT100-B2 & 37 & 20 & Twice & 3.93 & 2.32 \\
\hline
\end{tabular}

The students were not given instructions on how to construct their graph; some chose bar graphs while others chose line graphs. Figure 3 shows what the ideal graph would look like compared to what two of the students constructed.

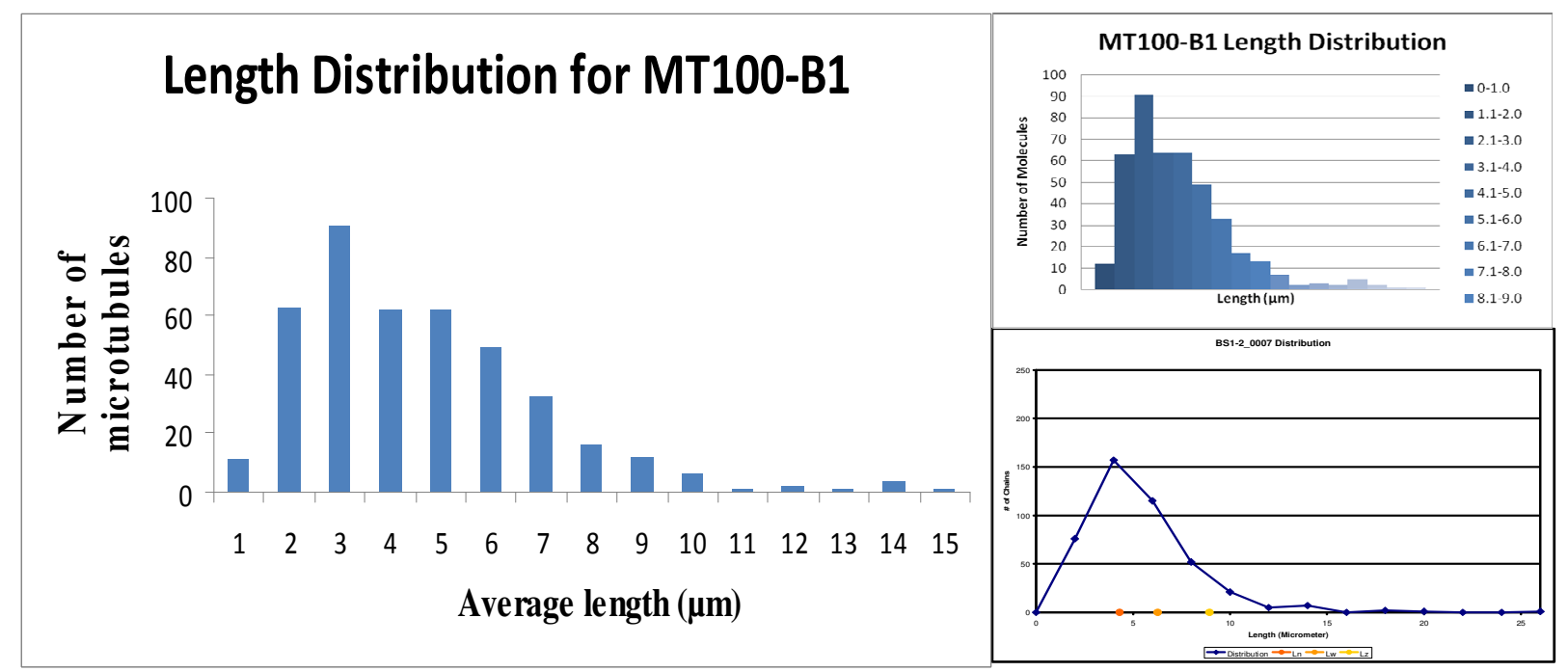

Figure 3: Length Distribution for Sample MT100-B1. The graph on the left was constructed by the Lab TA. The graph on the upper right was constructed by Student A, and the graph on the lower right was constructed by Student B. 
This exercise provided the students an opportunity to increase their understanding of polymer weight distribution. They could confirm their observations with their lecture notes and textbook information. They polymerized a polymer, characterized it by its molecular weight distribution and then looked at how polymerization and post-processing conditions varied the distribution.

\section{Assessment of Student Learning}

The students submitted a typed laboratory report. The achieved student learning is assessed qualitatively by the quality of the laboratory reports the students submit and by a post-lab discussion. Furthermore, the students were asked to complete a mini-evaluation of the Microtubule Laboratory at the completion of the course. Listed below are the questions asked accompanied with 2 students' responses, due to a small class size of 4 .

(1) Did this laboratory increase your knowledge about molecular weight distribution?
a. Student A: yes
b. Student B: yes

(2) Did this laboratory add to your understanding of biopolymers?
a. Student A: yes
b. Student B: yes

(3) Did this laboratory increase your knowledge of polymer processing

a. Student A: yes

b. Student B: yes

(4) How did this laboratory compare with the other laboratories? (i.e. better, worse, same, liked, dislike)

a. Student A: I think the lab was on par with other lab assignments. Microtubules are important in biomaterials, and I think it is important to be exposed to polymers such as these that exhibit different properties than typical synthetic polymers. I enjoyed the lab, although the analysis was time-consuming.

b. Student B: It was rather work intensive. The results came out clearly, so the time was well spent.

(5) What other concepts/topics of polymer properties, outside the two listed above, did you learn or were demonstrated in this laboratory?

a. Student A: I learned about microtubules and how they function, as well as got insight into possible methods of synthesis for these polymers. We also got to see 
how different factors such as polymerization time and temperature effect polymerization.

b. Student B: I learned about Brownian motion and polymer scizzion.

(6) What are some ways this laboratory could be improved?

a. Student A: Following better instruction when viewing the microtubules under the microscope.

b. Student B: Polymerize samples at two temps and four times, so one can look at trends. Also do the shear twice.

(7) Would you recommend this laboratory for this course? Give brief reason for your stance.

a. Student A: Yes, I would recommend this lab because it exposes polymer students to biopolymers. However, I would allow groups to split up the work when analyzing the lengths of the microtubules.

b. Student B: Yes, It was my favorite lab offered in the class. Using highly computerized microscopes and image software was really interesting.

(8) Any additional comments?

a. Student A: none

b. Student B: Optical microscopy is not really taught in the Materials Science \& Engineering Department.

\section{Discussion and Conclusion}

An opportunity to broaden the impact of this laboratory is its integration into a series of courses utilizing the Undergraduate Core Lab, which was established by the Honors Program at our institution. The goal of these courses, premiering in Spring 2008, is to increase life science knowledge of mathematical, chemistry, physics and engineering undergraduate students and to increase mathematical, chemical, physical and engineering knowledge of life sciences undergraduate students. In short the courses as well as the laboratories are meant to be transdisciplinary and interdisciplinary. The feasibility of transitioning the developed laboratory to an audience of about a thousand students will be assessed in the future by evaluating the financial and logistical requirements on a larger scale.

An important question is if and how the proposed laboratory can be conducted if research-grade equipment, such as a fluorescence microscope with high-power objectives, is not readily available. The equipment cost of the here utilized setup is about $\$ 40,000$ (new), an amount which justifies dedicated equipment only if many hundred students conduct this laboratory every year. As often, there are no simple solutions. The value of this paper is hopefully to present an 
opportunity, and stimulate the search for creative solutions by us and others. The next step could be to survey and test educational microscopes and determine which affordable improvements would lead to an acceptable imaging performance in the context of this laboratory. An alternative avenue worth pursuing may be the creation of a virtual laboratory, which combines multimedia techniques to recreate the experience of a remote-access user facility, such as the Pacific

Northwest National Laboratory's Environmental Molecular Sciences Laboratory ${ }^{10}$.

In conclusion, we have designed a microtubule-based laboratory which can be a valuable addition to the curriculum of MSE and BME students, specifically those specializing in polymers and biomaterials. This laboratory allowed students to visualize polymer properties (such as weight distribution) and effects of post-polymerization processes (such as shearing). The laboratory utilizes commercially available compounds and can fit into the time constraints of most class periods.

\section{Bibliography}

1. $\quad$ Hess, H.; Vogel, V. Journal of Biotechnology 2001, 82, (1), 67-85.

2. Hess, H.; Bachand, G. D.; Vogel, V. Chemistry - A European Journal 2004, 10, (9), 2110-2116.

3. $\quad$ Fischer, T.; Hess, H. Journal of Materials Chemistry 2007, 17, (10), 943-951.

4. $\quad$ Alberts, B.; Johnson, A.; Lewis, J.; Raff, M.; Roberts, K.; Walter, P., Molecular Biology of the Cell. Fourth Edition. 4 ed.; Garland: New York, 2002.

5. Howard, J., Mechanics of motor proteins and the cytoskeleton. Sinauer Associates Publishers: Sunderland, Mass., 2001; p xvi, 367.

6. $\quad$ Mitchison, T.; Kirschner, M. Nature 1984, 312, (5991), 237-242.

7. $\quad$ Schiff, P. B.; Fant, J.; Horwitz, S. B. Nature 1979, 277, (5698), 665-667.

8. Howard, J.; Hyman, A. A., Preparation of marked microtubules for the assay of the polarity of microtubulebased motors by fluorescence microscopy. In Methods in Cell Biology, Vol 39, 1993; Vol. 39, pp 105-113.

9. $\quad$ Chretien, D.; Metoz, F.; Verde, F.; Karsenti, E.; Wade, R. H. J Cell Biol 1992, 117, (5), 1031-40.

10. http://collaboratory.emsl.pnl.gov/information/remote_access.shtml. 


\section{Laboratory Handout}

\section{Objective:}

\section{Polymer Properties}

(1) Analyze microtubule length distribution as a function of:
a. Time
b. Temperature
c. Post polymerization shearing

(2) Observe Brownian motion

(3) Discuss some polymer processing issues
a. Extrusion
b. Chemical stability

\section{Materials:}

BRB80, 2.5mL

DMSO, $5 \mu \mathrm{L}$

GTP $(25 \mathrm{mM}), 5 \mu \mathrm{L}$

$\mathrm{MgCl}_{2}(100 \mathrm{mM}), 5 \mu \mathrm{L}$

Taxol, $30 \mu \mathrm{L}$

Aliquots of $20 \mu \mathrm{g}$ lyophilized, rhodamine-labeled tubulin (Cytoskeleton Inc.)

\section{Equipment:}

Nikon TE 2000s epi-Fluorescence Microscope

Nikon CCD camera

100x oil objective lens

Immersion oil

Glass coverslips

Eppendorff micropipette and pipet tips

Centrifuge tubes

Vortex

Water bath

Thermometer

Stopwatch or timer

Sharpie or other marking pen

30Gauge needles

\section{Method:}

MICROTUBULE POLYMERIZATION:

1. In a small centrifuge tube mix $43.5 \mu \mathrm{L}$ BRB80, $2.5 \mu \mathrm{L}$ DMSO, $2.0 \mu \mathrm{L}$ GTP and $2.0 \mu \mathrm{L}$ $\mathrm{MgCl}_{2}$.

2. Vortex the solution. 
3. Take four $20 \mu \mathrm{g}$ aliquot of rhodamine labeled tubulin and label each A-D.

4. Add $6.25 \mu \mathrm{L}$ of previous solution to each $20 \mu \mathrm{g}$ tubulin aliquot.

5. Allow the tubulin to polymerize at the appropriate time and temperature as indicated below:

\begin{tabular}{|l|l|l|}
\hline ID & Temperature $\left({ }^{\circ} \mathrm{C}\right)$ & Time $(\min )$ \\
\hline A & 25 & 20 \\
\hline B & 37 & 20 \\
\hline C & 37 & 10 \\
\hline D & 37 & 2 \\
\hline
\end{tabular}

6. While the tubulin is polymerizing, label 4 large centrifuge tubes A-D. In each large centrifuge tube add $490 \mu \mathrm{L}$ BRB80 and $5 \mu \mathrm{L}$ Taxol.

7. After the allotted polymerization time for each tubulin sample, transfer $5 \mu \mathrm{L}$ of the microtubules (polymerized tubulin) to the appropriately labeled large centrifuge tube. Label the tubes MT100, to the right of their letter IDs, example MT100-A.

\section{SHEARING:}

Take two small centrifuge tubes and label these B-1 and B-2. Take $5 \mathrm{~L}$ of MT100 solution from MT100-B and place in each of the two small centrifuge tubes. Shear the microtubules in tube B1 one time. Shear the microtubules in tube B-2 twice.

\section{IMAGING MICROTUBULES:}

1. Place $\sim 2 \mu \mathrm{L}$ of the MT100 solution on a glass slide and place a cover slip on top being careful to avoid air bubbles.

2. Image the microtubules under an epi-fluorescence microscope using the 100x oil objective.

3. Using the Andor iXon software and the Nikon CCD camera take and record 2-5 images of the microtubules.

4. Repeat for each sample. (Note: For MT100-B, B-1 and B-2 take 10 images each.)

\section{OPERATING THE MICROSCOPE:}

1. Turn on the X-Cite 120 Fluorescence Illumination System

2. Turn on the Lambda SC Smart Shutter Controller

3. Click open the Lambda SC software

a. In the smart shutter control area, uncheck trigger pulse and set status to open.

b. Set Port knob, on side of microscope, to setting 1 and focus microtubules with eyepiece.

c. Set Port knob to setting 5 .

d. On the Lambda menu screen set status to close and check trigger pulse and set it to high.

4. Open the Andor iXon software.

5. Click on Acquisition and select set up acquisition.

6. In the window, under the Set up CCD tab,
a. Set acquisition mode to kinetic
b. Set exposure time to $0.2 \mathrm{sec}$
c. Set kinetic series length to (number) of images desired 
d. Set kinetic cycle to $0.2 \mathrm{sec}$

7. In the Auto Save tab, select a path and file name to save images.

8. Exit menu screen.

9. To take images with camera, click on take image.

\section{$\underline{\text { Results: }}$}

1. Make a sketch of what you see when imaging the microtubules.

2. Using Image $J$ software, measure the lengths of the microtubules that are completely in the field of view. (Remember to take into account the pixel resolution of the camera and the magnification of the microscope's objective lenses.)

a. For the MT100-B, B-1 and B-2 samples, measure 1000 microtubules and create a length distribution

b. For other samples, measure 100 microtubules and calculate an average length; be sure to include measurement errors

3. Graph your results:

a. Average microtubule length as a function of polymerization temperature

b. Microtubule length as a function of polymerization time

c. Microtubule length as a function of shearing

\section{Discussion:}

1. In Image J, the data from the images are recorded as pixels. How did you convert these to appropriate length-scale units?

2. With what accuracy can you measure the length of the microtubules?

3. How is error measured? What is the error in your measurements?

4. How does average microtubule length vary as a function of polymerization temperature?

5. How does polymerization time and shearing affect microtubule length distribution?

6. What is Brownian motion? Did you observe Brownian motion while imaging the microtubules? Explain. 